\title{
Painkiller concerns grow ahead of new guidelines
}

In 1887, German pharmacologist Joseph von Mering determined that a coal tar-derived chemical called paracetamol was potentially dangerous because it caused changes to hemoglobin in the blood that might deprive tissues of oxygen. Paracetamol (better known as acetaminophen in the US) spent the next six decades in pharmaceutical exile. But, in the early 1950s, further research showed that Mering's findings were incorrect, possibly owing to tainted sources of the drug: the compound could deliver pain-reducing properties with minimal side effects.

Since that redemption, paracetamol has enjoyed an unparalleled reputation for safety among over-the-counter pain relievers. It remains one of the most commonly used drugs in the world, with nearly 28 billion doses sold in the US in 2008, according to the US Food and Drug Administration (FDA). However, as evidence of its risk grows, regulators are now poised to make changes to reign in use of the drug.

Paracetamol "is still extraordinarily safe when used correctly," says Lewis Nelson, a toxicologist who served as the acting chairperson for an FDA advisory committee panel that, in June, heard evidence on the dangers of paracetamol. "But it's not candy. It's a potent drug. The fact that it's not always given due consideration is causing problems - some of which we've only recently learned."

The most substantial concern is liver toxicity. The panel heard evidence that, between 1998 and 2003, paracetamol was the leading cause of acute liver failure in the US, although half of the cases were caused by people intentionally overdosing.

Ultimately, the panel suggested that the FDA implement ten restrictions on the use of paracetamol, most of which involved reducing the size of the packaging and dosages. This parallels regulations that have been in place in the UK since 1998, requiring over-the-counter packages of paracetamol to contain no more than 16 tablets with a cumulative maximum of $8 \mathrm{~g}$ of the drug.

The FDA is currently reviewing these recommendations, and should propose new regulations in early 2010. However, these would be targeted at reducing liver-toxicity issues only.

"It's important to understand that, even though this drug is a hundred years old, there are still a lot of mechanisms that aren't completely understood," says Matt Perzanowski, an asthma researcher at the
Mailman School of Public Health at Columbia University in New York.

Perzanowski's research has found that prenatal exposure to larger-than-suggested doses of paracetamol substantially increases the chance of breathing problems in children (Thorax, doi:10.1136/thx.2009.121459; 2009). The work dovetails with a November review of 19 studies (covering more than 425,000 participants), which found that, overall, regular acetaminophen users were $63 \%$ more likely to have asthma than nonusers (Chest, 136, 13161323; 2009).

Other research has suggested that the pain reliever might increase the risk of birth defects, although these results remain controversial.

A study conducted in the Czech Republic, meanwhile, showed that paracetamol used to reduce fever associated with infant vaccinations impaired the children's immune response to those vaccinations (Lancet, 374, 1339-1350; 2009).

Researchers are still not certain why this happens or exactly how much this effect diminishes the immunity derived from the vaccines, says Robert Chen, who, with colleagues

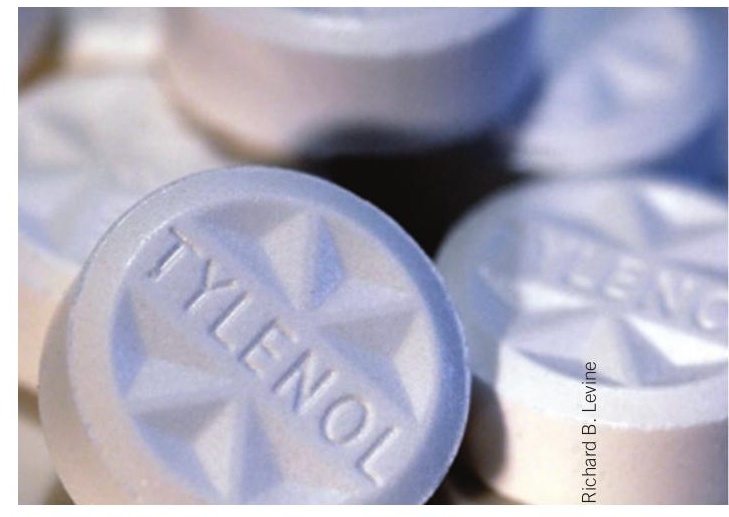

A splitting headache: Breaking from pain relievers

at the US Centers for Disease Control and Prevention, wrote a review of the study in the same issue (Lancet, 374, 1305-1306; 2009).

"Children don't usually need treatment for their fevers after vaccinations," Chen says. "I think sometimes it's more to help the parents' state of mind than the children's health. But, acetaminophen isn't a benign substance-the children are being given something that really affects their health."

Stu Hutson, Gainesville, Florida

\section{Caution urged on tobacco research}

In June 2009, the US Food and Drug Administration (FDA) received the authority to regulate tobacco. The following month, the FDA asked for public comments on the new regulatory process, and, in December, the American Association for Cancer Research (AACR) responded with advice on expediting scientific research on tobacco.

To jump-start new research, the AACR published nine research reviews in Cancer Epidemiology, Biomarkers \& Prevention last month that detail strategies and trial designs that the agency could use to assess harm and risk.

Among the nine papers is a study addressing the limitations of smoking machine tests and toxicology assays that use nonhuman cells. The authors warn that extrapolating from these studies to determine the risks to humans can be problematic. Another study reviews methods used to determine addiction potential and how these approaches might apply to 'modified-risk tobacco products', a new class of tobacco products that the FDA will assess and regulate.

Relative to the quantity of safety research that exists for drugs safety, solid tobacco research is relatively scarce, according to Peter Shields, deputy director of the Lombardi Comprehensive Cancer Center at Georgetown Medical Center in Washington, DC, and editor of the reviews. Much of what exists comes from tobacco companies themselves and is either outdated or unreliable.

The FDA "will review the information submitted by AACR to our public docket regarding best practices on clinical trail and design," says agency spokeswoman Kathleen Quinn.

But Philip Landrigan, head of preventive medicine at the Mount Sinai School of Medicine in New York, cautions that "there needs to be a proper balance between research and action."

"While I don't want to discourage research, the FDA has all it needs to know now to take decisive action to reduce risks of tobacco," he added.

Vicki Brower, New York 